\title{
THE FREE LATTICE-ORDERED GROUP OVER A NILPOTENT GROUP
}

\author{
MICHAEL R. DARNEL
}

(Communicated by Warren J. Wong)

\begin{abstract}
We show that the free lattice-ordered group over a finitely generated torsionfree nilpotent group is $l$-solvable of some finite rank.
\end{abstract}

\section{INTRODUCTION}

Conrad [C] showed that for a partially ordered group $G$, a free lattice-ordered group over $G$ exists if and only if the partial order of $G$ is the intersection of right orders of $G$. In particular, taking $G$ to be trivially ordered, a free lattice-ordered group $F(G)$ over the group $G$ exists if and only if $G$ can be right-ordered: a total order $\leq$ exists on $G$ such that if $g \leq h$ in $G$, then for any $x \in G, g x \leq h x$.

The construction of $F(G)$ is easy to describe. Let $\left\{\leq_{\lambda}\right\}_{\Lambda}$ be the set of all right orders of $G$. For each $\leq_{\lambda}, G$ acts (by multiplication on the right) as a group of order-preserving permutations of the chain $\left(G, \leq_{\lambda}\right)$. So by way of the right regular representation, $G$ can be embedded into $\mathscr{A}\left(G, \leq_{\lambda}\right)$, the $l$-group of all order-preserving permutations of the chain $\left(G, \leq_{\lambda}\right) . F(G)$ is then the l-subgroup of $\prod_{\Lambda} \mathscr{A}\left(G, \leq_{\lambda}\right)$ generated by the "long constants" of $G: g \rightarrow\left(\ldots, \bar{g}_{\lambda}, \ldots\right)$. More useful in the following discussion is that if $G_{\lambda}^{*}$ is the $l$-subgroup of $\mathscr{A}\left(G, \leq_{\lambda}\right)$ generated by the right regular representation of $G$, then $F(G)$ is the $l$-subgroup of $\Pi_{\Lambda} G_{\lambda}^{*}$ generated by the long constants of $G$. Thus if we can demonstrate that each $G_{\lambda}^{*}$ is in a variety of lattice-ordered groups, then $F(G)$ must be as well.

Very little is known at this time about what characteristics of $G$ carry over to $F(G)$. It is well known that if $G$ is abelian, then $F(G)$ is too. Darnel and Glass [DG] proved that if $G$ is a torsionfree nilpotent group of class 2 (hereafter referred to as nil-2) generated by $m$ elements, then $F(G)$ is $l$-solvable of rank $\left(\begin{array}{c}m \\ 2\end{array}\right)+1$ but may not be nilpotent.

Received by the editors October 1, 1988 and, in revised form, March 12, 1990; the results of this paper were presented at the Curacao Conference on Ordered Algebraic Structures, August 1988, Curacao, and appear without proof in the proceedings of that conference [D1].

1980 Mathematics Subject Classification (1985 Revision). Primary 06F20.

This research was supported in part by a Summer Faculty Fellowship, Indiana University at South Bend, 1987. 
We will make use of the following theorem which gives a characterization for when the free lattice-ordered group over $G$ is normal-valued. First recall that a lattice-ordered group $H$ is normal-valued if for any $g, h \in H$, $|x||y| \leq\left|y^{2}\right|\left|x^{2}\right|$. This is equivalent to the condition $|[x, y]| \ll|x| \bigvee|y|$ and to the condition: if $g, h \ll k$, then $g h \ll k$. These three conditions are also equivalent for right-ordered groups, and a right-ordered group satisfying any (and hence all) of the conditions is called a Conrad right-ordered group, more commonly known as a $c$-group. The right order is then called a $c$-order.

Theorem 1 [GHR]. The free lattice-ordered group over a group $G$ is normalvalued if and only if every right order of $G$ is a c-order.

(What was actually shown in [GHR] is that if $(G, \leq)$ is a $c$-group, then $G^{*}$ in $\mathscr{A}(G, \leq)$ is normal-valued. Theorem 1 is then an easy consequence of this result.)

\section{THE $l$-SUBGROUP GENERATED BY A $c$-ORDERED PERMUTATION GROUP}

Let $G$ be a lattice-ordered group and $A \subseteq G$ be a subgroup of $G .\langle A\rangle$ will denote the $l$-subgroup of $G$ generated by $A$. For any $a \in\langle A\rangle$, there exist finite sets $I$ and $J$ and $\left\{a_{i j}\right\}_{i \in I, j \in J} \subseteq A$ such that $a=\bigvee_{I} \bigwedge_{J} a_{i j}=\bigwedge_{J} \bigvee_{f \in I^{J}} a_{f(j), j}$. $G(A)$ will denote the convex $l$-subgroup of $G$ generated by $A$; then $G(A)=$ $\{g \in G:|g| \leq|a|$ for some $a \in A\}$. As in the introduction, if $(G, \leq)$ is a right-ordered group, then $G^{*}$ is the $l$-subgroup of $\mathscr{A}(G, \leq)$ generated by the representation of $G$ acting on the chain $(G, \leq)$ by right multiplication.

Lemma 2. Let $G$ be a lattice-ordered group and $A \subseteq B$ be subgroups of $G$ such that $A$ is normal in $B$ and $\langle B\rangle=G$. Then $G(A)$ is normal in $G$.

Moreover, if for any $b \in B \backslash A, b$ is either positive or negative in $G$ and if for any $e<b \in B \backslash A$ and any $a \in A,|a| \ll b$, then $G$ is a lex extension of $G(A)$ and $G(A)=\langle A\rangle$.

Proof. Let $b \in B$ and $h \in\langle A\rangle$; then $h=\bigvee_{I} \bigwedge_{J} a_{i j}$ as above. So $b^{-1} h b=$ $\bigvee_{I} \wedge_{J}\left(b^{-1} a_{i j} b\right)$ which is clearly in $\langle A\rangle$. So $B$ normalizes $\langle A\rangle$ and hence normalizes $G(A)$. Since the normalizer of a convex $l$-subgroup is an $l$-subgroup [D2], $G=\langle B\rangle$ normalizes $G(A)$.

For the second part, we will show that any $g \in G$ is either an element of $\langle A\rangle$ or can be written in the form $z b$, where $z \in\langle A\rangle$. and $b \in B \backslash A$. Now $g=\bigwedge_{I} \bigvee_{J} b_{i j}$, where $b_{i j} \in B$ for all $i$ and $j$. We will first assume that $I$ is a single element and so $g=\bigvee_{J} b_{j}$.

Let $J^{\prime}=\left\{j \in J: b_{j} \notin A\right\}$. If $J^{\prime}$ is empty, then $g \in\langle A\rangle$. So assume $J^{\prime}$ is not empty. Now if $b_{i_{1}}$ and $b_{i_{2}}$ are in different cosets of $A$, then $b_{i_{1}} b_{i_{2}}^{-1} \notin A$ and so is either positive or negative. Clearly then $b_{i_{1}} \vee b_{i_{2}}$ is the larger of the two. Also clear is the fact that if $b_{i_{1}}$ is larger than $b_{i_{2}}$ and $b_{i_{3}}$ is in the same $A$-coset as $b_{i_{1}}$, then $b_{i_{3}}>b_{i_{2}}$. Thus $\bigvee_{J} b_{j}$ is the join of those $b_{j}$ 's in the 'highest' coset of $A$. Now if $b_{i_{1}}$ and $b_{i_{2}}$ are in this coset, then $b_{i_{2}}=a b_{i_{1}}$ and 
so $b_{i_{1}} \vee b_{i_{2}}=(e \vee a) b_{i_{1}}$. So clearly $\bigvee_{J} b_{j}$ is of the form $z b$, where $z \in\langle A\rangle$ and $b \in B$. Note that if $b \in A$, then this join is in $\langle A\rangle$.

Next consider $\bigwedge_{I} z_{i} b_{i}$, where $z_{i} \in\langle A\rangle$ and $b_{i} \in B$. Once again, if the $A$ coset of $b_{i_{1}}$ is not that of $b_{i_{2}}$, then $b_{i_{1}}$ and $b_{i_{2}}$ are comparable and so $z_{i_{1}} b_{i_{1}}$ is comparable to $z_{i_{2}} b_{i_{2}}$. Thus we need consider only those $z_{i} b_{i}$ 's is the 'lowest' coset of $A$; call this subset $I^{\prime}$. So $\bigwedge_{I^{\prime}} z_{i} b_{i}$ is of the form $z b$, where $z \in\langle A\rangle$ and $b \in B$.

Now let $g \in G \backslash[G(A)]$; then $g=z b$ where $z \in\langle A\rangle$ and $b \in B \backslash A$. Then $b$ is either positive or negative and since $|b| \gg|z|, g$ is positive or negative as $b$ is. Clearly anything in the $G(A)$-coset of $g$ is also positive or negative as $g$ is. So $G$ is a lex extension of $G(A)$. Equally clear now is that $G(A)=\langle A\rangle$.

Proposition 3. Let $\left(G, \leq_{r}\right)$ be a right-ordered group and let $K$ be the convex subgroup of $G$ generated by the derived group $G^{(1)}$. If $K \neq G$, then $G^{*}$ is a lex extension of $K^{*}$.

Proof. For any $g \in G$, let $\bar{g}$ denote the order-preserving permutation of the chain $\left(G, \leq_{r}\right)$ determined by multiplying elements on the right by $g$.

Let $e<_{r} g \in G \backslash K$. If $\bar{g} \ngtr e$ in $G^{*}$, there exists $\alpha \in G$ such that $\alpha g<_{r} \alpha$. But then $\alpha g \alpha^{-1} g^{-1}<_{r} g^{-1}<_{r} e$, which implies that $g^{-1}$ and hence $g$ is in $K$. So $e<_{r} g \in G \backslash K$ implies that $\bar{g}>e$ in $G^{*}$.

Now suppose that for some $k \in K$ and $e<_{r} g \in G \backslash K,|\bar{k}| \nless \bar{g}$. Then there exists $\alpha \in G$ such that $\alpha g<_{r} \alpha k$. Since $K$ is normal in $G, \alpha k=k_{1} \alpha$ and so $\alpha g<_{r} k_{1} \alpha$ implies that $\alpha g \alpha^{-1} g^{-1}<_{r} k_{1} g^{-1}<_{r} e$, a contradiction. So $\bar{g} \gg|\bar{k}|$ for all $k \in K$. Lemma 2 now applies.

Corollary 4. Let $G$ be a finitely generated group and $\leq_{r}$ be a c-ordering of $G$. Let $K$ be the convex subgroup of $G$ generated by the commutator subgroup. Then in $\mathscr{A}\left(G, \leq_{r}\right), G^{*}$ is a lex extension of $K^{*}$.

Proof. Let $\left\{a_{1}, \ldots, a_{n}\right\}$ be generators of $G$. By using inverses if necessary, we can assume that $a_{1}>_{r} \cdots>_{r} a_{n}>_{r} e$. Then clearly $a_{1}$ is infinitely greater than any commutator and so $K \neq G$.

\section{THE FREE LATTICE-ORDERED GROUP OVER A NILPOTENT GROUP}

We will call a lattice-ordered group $l$-solvable of rank $n$ if there exists a chain of convex subgroups

$$
(e) \triangleleft A_{0} \triangleleft A_{1} \triangleleft \cdots \triangleleft A_{n}=G
$$

such that each quotient $A_{i+1} / A_{i}$ is abelian. Smith [S] pointed out that while $l$-solvability of rank $n$ implies solvability of rank $n$, the converse is not true. In [GHM], it was shown that $w r^{n} \mathscr{Z}$, the iterated ordered wreath product of the ordered group $\mathscr{Z}$ of integers with itself $n$ times, generates the variety of $l$-solvable lattice-ordered groups of rank $n$. 
Finally, let $G$ be a finitely generated torsionfree nilpotent group. Then each subgroup $Z_{i}(G)$ of the ascending central series is a pure (or isolated) subgroup and so, for any $i, Z_{i+1} G / Z_{i}(G)$ is a finitely generated torsionfree abelian group and so is free. Thus there exists a central series

$$
(e)=A_{0} \triangleleft A_{1} \triangleleft \cdots \triangleleft A_{n}=G
$$

such that each $A_{i+1} / A_{i}$ is free abelian of rank one. Hirsch [H] proved that any two such central series must have a common length (which is the minimum length of a central series with cyclic factors) called the Hirsch length or Hirsch number of the group. (The interested reader should also see [B, p. 184], or [Ha, Chapter 10].)

Theorem 5. Let $G$ be a finitely generated torsionfree nilpotent group of Hirsch length $n$. Then the free lattice-ordered group $F(G)$ over $G$ is l-solvable of rank at most $n$.

Proof. We of course induct on $n$.

If $n=1$, then $G$ is abelian and so $F(G)$ is abelian. So suppose $n>1$ and that the theorem is true for all finitely generated nilpotent groups whose Hirsch length $k$ is less than $n$.

Let $\leq_{r}$ be a right order of $G$ and let $K$ be the convex subgroup of $G$ generated by the commutators. Rhemtulla [R] proved that any right order of a nilpotent group must be a $c$-order. By Corollary $4, G^{*}$ is a lex extension of $K^{*}$.

Now $G / K$ is free abelian and so the Hirsch length of $K$ is less than that of $G$. By induction, $F(K)$ is $l$-solvable and so $K^{*}$ is $l$-solvable because $K^{*}$ is an $l$-homomorphic image of $F(K)$. Since $G^{*} / K^{*}$ is abelian, $G^{*}$ is $l$-solvable of rank at most $n$.

The above theorem shows that the free lattice-ordered group over a finitely generated nilpotent group is $l$-solvable of rank less than or equal to the Hirsch length. As mentioned above, Darnel and Glass [DG] proved that the free latticeordered group over a torsionfree nil-2 group generated by $m$ elements is $l$ solvable of rank $\left(\begin{array}{c}m \\ 2\end{array}\right)+1$. For a comparison of these results, it is instructive to examine the free nil-2 groups of finite rank.

Let $F_{n}$ be the free nil-2 group on free generators $\left\{a_{1}, \ldots, a_{n}\right\}$. Since $F_{n}$ is free, for any $1 \leq i<j \leq n$, the commutator $\left[a_{i}, a_{j}\right] \neq e$. Thus the center $Z\left(F_{n}\right)$ is a free abelian group on the set of commutators $\left\{\left[a_{i}, a_{j}\right]: 1 \leq i<j \leq\right.$ $n\}$. Note also that any element of $F_{n}$ can be written uniquely in the form

$$
a_{1}^{m_{1}} a_{2}^{m_{2}} \cdots a_{n}^{m_{n}}\left[a_{1}, a_{2}\right]^{m_{12}} \cdots\left[a_{n-1}, a_{n}\right]^{m_{n-1, n}} .
$$

Now the Hirsch length of $F_{n}$ is $n+\left(\begin{array}{l}n \\ 2\end{array}\right)$, which is clearly greater than the bound of Darnel and Glass. But we can take a homomorphic image $H$ of $F_{n}$ in which $\left[a_{i}, a_{j}\right]=e$ for $1<i<j \leq n$. The Hirsch length of $H$ is then $2 n-1$, which is less than the bound given by Darnel and Glass.

The following theorem improves both bounds. 
Theorem 6. Let $G$ be a finitely generated torsionfree nil-2 group and let $n$ be the minimal number of generators for $G$. Then the free lattice-ordered group $F(G)$ over $G$ is l-solvable of rank at most $n$.

Proof. Let $a_{1}, a_{2}, \ldots, a_{r}$ be elements of $G$ such that their cosets are free generators of $G / Z(G)$; let $A$ be the subgroup of $G$ generated by $\left\{a_{1}, \ldots, a_{r}\right\}$. If $A \neq G$, then there exist elements $b_{r+1}, \ldots, b_{n}$ in $Z(G)$ such that, letting $B$ be the subgroup of $G$ generated by $\left\{b_{r+1}, \ldots, b_{n}\right\}, G$ is the direct sum of $A$ and $B$.

Note that if $r \leq 1$, then $G$ is abelian and so $F(G)$ is abelian. Clearly this is the case if $n=1$.

So suppose $n>1$ and that $r>1$; further suppose that if $A$ is generated by $k<r$ generators, the free lattice-ordered group over $A \times B$ is $l$-solvable of rank $k$.

Let $\leq_{r}$ be a right order of $G$ and let $K$ be the convex subgroup of $G$ generated by the commutators. If $K$ (and hence $K^{*}$ ) is abelian, then $G^{*} / K^{*}$ is abelian and so $G^{*}$ is $l$-solvable of rank 2 . So suppose that $K$ is not abelian. Then $A K / K$ is a free abelian group of rank $m<r$. So we can assume, by rearranging and taking appropriate combinations if necessary, that $a_{1}, a_{2}, \ldots, a_{m}$ are not in $K$ while $a_{m+1}, \ldots, a_{r}$ are in $K$. Similarly, we can choose free generators $b_{r+1}, \ldots, b_{n}$ of $B$ such that there exists $r+1 \leq s \leq n$ so that the set $\left\{K b_{r+1}, \ldots, K b_{s}\right\}$ freely generates $B K / K$ and for any $s<j \leq n, b_{j} \in K$. Since $G$ is the direct sum of $A$ and $B, G / K$ is the direct sum of $A K / K$ and $B K / K$; thus in $G / K$, the set $\left\{K a_{1}, \ldots, K a_{m}, K b_{r+1}, \ldots, K b_{s}\right\}$ is a free set of generators.

However, $G^{*} / K^{*}$ is then a free abelian group with free generators $\left\{K^{*} \bar{a}_{1}, \ldots\right.$, $\left.K^{*} \bar{a}_{m}, K^{*} \bar{b}_{r+1}, \ldots, K^{*} \bar{b}_{s}\right\}$. Note that $K$ is generated by $\left\{a_{m+1}, \ldots, a_{r}\right\} \cup$ $\left\{\left[a_{i}, a_{j}\right]: 1 \leq i \leq m, i+1 \leq j \leq n\right\} \cup\left\{b_{s+1}, \ldots, b_{n}\right\}$. However, the elements of the last two sets generate a free abelian group. So by induction $F(K)$ is $l$-solvable of rank at most $r-m$, and thus $K^{*}$ is as well. So $G^{*}$ is $l$-solvable of rank $r-m+1$ which is less than $n$.

The bound in Theorem 6 is the best possible. Again consider $F_{n}$, the free nil-2 group on free generators $a_{1}, \ldots, a_{n}$. One can build a total order on $F_{n}$ since every element can be written uniquely in the form

$$
a_{1}^{m_{1}} a_{2}^{m_{2}}\left[a_{1}, a_{2}\right]^{m_{12}} a_{3}^{m_{3}} \cdots\left[a_{n-2}, a_{n-1}\right]^{m_{n-1, n}} a_{n}^{m_{n}}\left[a_{1}, a_{n}\right]^{m_{1, n}} \cdots\left[a_{n-1}, a_{n}\right]^{m_{n-1, n}} .
$$

Ordering lexicographically from the left defines a two-sided order on $F_{n}$ under which $F_{n}$ is $l$-solvable of rank $n$ but not rank $n-1$.

But a better result is possible. We can actually embed the iterated small wreath product $w r^{n} \mathscr{Z}$ with its usual lattice ordering into the free lattice-ordered group over $F_{n}$. (This was shown in [DG] when $n=2$.) 


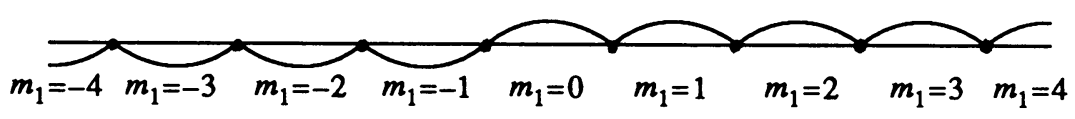

FIGURE 1. Graph of $\bar{a}_{2}$

To do this, we can alternatively write every element of $F_{n}$ uniquely in the form

$a_{1}^{m_{1}}\left[a_{1}, a_{2}\right]^{m_{12}} \cdots\left[a_{1}, a_{n}\right]^{m_{1 n}} a_{2}^{m_{2}}\left[a_{2}, a_{3}\right]^{m_{23}} \cdots\left[a_{2}, a_{n}\right]^{m_{2 n}} \cdots\left[a_{n-1}, a_{n}\right]^{m_{n-1, n}} a_{n}^{m_{n}}$.

The positive cone of a right order $\leq_{r}$ on $F_{n}$ can then be defined lexicographically from the left by nonnegative powers. For future reference, let $H$ be the convex subgroup of $\left(F_{n}, \leq_{r}\right)$ generated by $a_{2}$. As before, we embed $F_{n}$ into $\mathscr{A}\left(F_{n}, \leq_{r}\right)$ by its right regular action.

It is then easy to see that $\bar{a}_{1}$ is positive in $F_{n}^{*}$, as is any element of the form $\left[\bar{a}_{i}, \bar{a}_{j}\right]$, when $i<j$. However, $\alpha \bar{a}_{2}>_{r} \alpha$ if and only if, when $\alpha$ is placed into the above standard form, $m_{1} \geq 0$.The action of $\bar{a}_{2}$ is shown in Figure 1.

It is also easy to verify that $\alpha\left(\overline{a_{1}^{-1} a_{2}^{-1} a_{1}}\right)>_{r} \alpha$ if and only if, referring again to the standard form of $\alpha, m_{1} \leq 0$. Thus the support of $\left(\bar{a}_{2} \wedge \bar{a}_{1}^{-1} \bar{a}_{2}^{-1} \bar{a}_{1}\right) \vee \bar{e}$ is $\left\{\alpha: m_{1}=0\right\}$. Furthermore, for all such $\alpha, \alpha\left(a_{1}^{-1} a_{2}^{-1} a_{1}\right)>_{r} \alpha a_{2}$, and so $\left(\bar{a}_{2} \wedge \bar{a}_{1}^{-1} \bar{a}_{2}^{-1} \bar{a}_{1}\right) \vee \bar{e}$ is just the component $c_{2}$ of $\bar{a}_{2}$ on $\left\{\alpha: m_{1}=0\right\}$. If for $2<i \leq n$, we further define $c_{i}$ to be the component of $a_{i}$ with support $\left\{\alpha: m_{1}=0\right\}$, then the $l$-subgroup of $F_{n}^{*}$ generated by $\left\{c_{2}, \ldots, c_{n}\right\}$ is $l$ isomorphic to the $l$-subgroup $H^{*}$ generated by the action of $H$ in $\mathscr{A}\left(H, \leq_{r}\right)$ and so by induction contains a copy of $w r^{n-1} \mathscr{Z}$.

But since for any $n \neq 0, \bar{a}_{1}^{-n} c_{2} \bar{a}_{1}^{n} \wedge c_{2}=e, F_{n}^{*}$ must contain a copy of $w r^{n} \mathscr{Z}$. So we have proved:

Proposition 7. If $F_{n}$ is the free nil-2 group of rank $n$, then $F\left(F_{n}\right)$ generates the l-solvable variety of rank $n$.

As pointed out in [DG], this shows that the free lattice-ordered group over a nilpotent group is not nilpotent if the group is nonabelian.

\section{REFERENCES}

[B] K. S. Brown, Cohomology of groups, Graduate Texts in Math., vol. 87, Springer-Verlag, 1982.

[C] P. F. Conrad, Free lattice-ordered groups, J. Algebra 16 (1970), 191-203.

[D1] M. R. Darnel, Recent results on the free lattice-ordered group over a right-orderable group, Proc. Curacao Conf. on Ordered Algebraic Structures, Kluwer, 1989, pp. 51-57.

[D2] _ Special-valued l-subgroups and abelian covers, Order 4 (1987), 191-194.

[DG] M. R. Darnel and A. M. W. Glass, Commutator relations and identities in lattice-ordered groups, Michigan Math. J. 36 (1989), 203-211.

[GHM] A.M.W. Glass, W. C. Holland, and S. McCleary, The structure of l-group varieties, Algebra Universalis 10 (1980), 1-20. 
[GHR] A.M.W. Glass, H. Hollister, and A. Rhemtulla, Right orderings versus lattice-orderings, Notices Amer. Math. Soc. 2 (1978), A-222.

[Ha] M. Hall, Jr., The theory of groups, Chelsea, 1976.

[H] K. A. Hirsch, Über lokal-nilpotente gruppen, Math. Zeit. 63 (1955), 290-294.

[R] A. Rhemtulla, Right-ordered groups, Canad. J. Math. 24 (1972), 891-895.

[S] J. E. Smith, Solvable and l-solvable l-groups, Algebra Universalis 18 (1984), 106-109.

[W] E. Weinberg, Free lattice-ordered abelian groups, Math. Ann. 151 (1963), 187-199.

Department of Mathematics, Indiana University at South Bend, South Bend, Indiana 46634 